\title{
Triboelectrification of pharmaceutical powders by particle impact.
}

\section{AUTHOR(S):}

Watanabe, Hideo; Ghadiri, Mojtaba; Matsuyama, Tatsushi; Ding, Yu Long; Pitt, Kendal G; Maruyama, Hiroyuki; Matsusaka, Shuji; Masuda, Hiroaki

\section{CITATION:}

Watanabe, Hideo ...[et al]. Triboelectrification of pharmaceutical powders by particle impact.. International journal of pharmaceutics 2007, 334(1-2): 149-155

\section{ISSUE DATE:}

2007-04-04

URL:

http://hdl.handle.net/2433/194296

\section{RIGHT:}

(c) 2006 Elsevier B.V.: NOTICE: this is the author's version of a work that was accepted for publication in International Journal of Pharmaceutics. Changes resulting from the publishing process, such as peer review, editing, corrections, structural formatting, and other quality control mechanisms may not be reflected in this document. Changes may have been made to this work since it was submitted for publication. A definitive version was subsequently published in International Journal of Pharmaceutics, 334(1-2) 149-156, 2004, doi:10.1016/j.jpharm.2006.11.005; This is not the published version. Please cite only the published version.; この論文は出版社版でありません。引用の際には出版社版 をご確認ご利用ください。 
Title:

Triboelectrification of pharmaceutical powders by particle impact

Authors:

Hideo Watanabe ${ }^{1}$, Mojtaba Ghadiri ${ }^{1 *}$, Tatsushi Matsuyama ${ }^{2}$, Yu Long Ding ${ }^{1}$, Kendal G. Pitt ${ }^{3}$, Hiroyuki Maruyama ${ }^{4}$, Shuji Matsusaka ${ }^{4}$, Hiroaki Masuda ${ }^{4}$

Affiliations:

1. Institute of Particle Science and Engineering, University of Leeds, Leeds LS2 9JT, UK

2. Faculty of Engineering, Soka University, Tokyo, 192-8577 Japan

3. Merck Sharp and Dohme, Hoddesdon, Hertfordshire EN11 9BU, UK

4. Department of Chemical Engineering, Kyoto University, Kyoto, 615-8510 Japan

Corresponding authors:

Prof. Mojtaba Ghadiri, Institute of Particle Science and Engineering, University of Leeds, Leeds LS2 9JT, UK

Tel: + 44 (0)113 343 2406, Fax: +44 (0)113 343 2405,

E-mail: M.Ghadiri@leeds.ac.uk 
Abstract: (less than 200 words):

Pharmaceutical powders are very prone to electrostatic charging by colliding and sliding contacts with walls and other particles. In pharmaceutical formulation processes, particle charging is often a nuisance and can cause problems in the manufacture of products, such as affecting powder flow, and reducing fill and dose uniformity. For a fundamental understanding of the powder triboelectrification, it is essential to study charge transfer due to a single contact of a particle with a target plane under well-defined physical, mechanical and electrical conditions. In this study, charge transfer due to a single impact of a particle against a stainless steel target was measured for $\alpha$-lactose monohydrate, aspirin, sugar granules and ethylcellulose. The amount of transferred charge is expressed as a function of impact velocity and impact angle as well as the initial charge. The maximum contact area during impact between a particle and a target plane is estimated by an elastic-plastic deformation model. It is found that the transferred charge is a linear function of the contact area. For a given material there is an initial particle charge for which no charge transfer occurs due to impact. This is found to be independent of impact velocity and angle, and is hence viewed as a characteristic property, which is related to the contact potential difference and tribo-electric series of the sample powders.

Keywords: (up to 6 words or short phrases)

Triboelectrification; Charge transfer; Particle impact; Equilibrium charge; Triboelectric series; Contact potential difference. 


\section{Introduction}

Pharmaceutical powders are very prone to electrostatic charging by colliding and sliding contacts with walls and other particles because they normally have a high resistivity, which prevents the transferred charge from leaking back (Grosvenor and Staniforth, 1996). The charging process is often described as triboelectrification or tribo-charging since sliding/frictional contacts are invariably involved (Bailey, 1984). The electrostatic forces acting on charged pharmaceutical particles may dominate in adhesion and deposition of the particles to walls, especially in the case of fine particle systems, such as dry powder inhalers (DPI) (Bailey, 1997, 1998; Balachandran et al., 1997). In the pharmaceutical industry, particle charging is often a nuisance and can cause problems in the manufacture of products, such as affecting powder flow, and reducing fill and dose uniformity (Staniforth, 1994). However, there are also cases where the electrostatic forces are used advantageously for control of drug particles such as for a DPI (Balachandran et al., 1997; Byron et al., 1997) or mixing of ordered powders (Staniforth and Rees, 1981a, b). In both cases it is necessary to control the charging propensity of pharmaceutical powders.

Triboelectrification of pharmaceutical powders has been investigated using a cyclone separator (Stainforth, 1994a; Eilbeck et al., 1999, 2000; Rowley, 2001; Rowley and Mackin, 2003), a DPI device (Murtomaa et al., 2004) or flowing through a glass pipe (Murtomaa et al., 2000, 2002). The charging processes in such systems are very complicated, as they include multiple particle-wall and inter-particle interactions and space charge effects, and hence are difficult to analyse. The knowledge obtained from these multiple particulate systems is useful for comparative evaluations, but lacks generality. It is far simpler, and in fact essential, to study charge transfer due 
to a single contact by a particle with a target plane for a fundamental understanding of the elementary processes. Matsuyama and Yamamoto (2006) reviewed the single particle testing method and termed it "impact charging test”. Results are reported in the literature for polymer particles of few millimetres in diameter (Masui and Murata 1983; Yamamoto and Scarlett 1986; Matsuyama and Yamamoto 1994, 1995), with $30 \mathrm{~mm}$ rubber balls (Matsusaka et al., 2001), with $550 \mu \mathrm{m}$ glass beads (Ema et al., 2003) and with several hundred micrometres polymer particles (Matsuyama et al., 2003). In these previous studies, spherical particles were mainly tested. We have recently developed a new impact charging test rig that enables testing of particles with arbitrary shapes and sizes smaller than hitherto tested (Watanabe et al., 2006). The results show that the tests characterise two important parameters: impact charge, $\Delta q_{\mathrm{o}}$, which is the amount of charge transferred due to a single impact for zero initial charge and equilibrium charge, $\mathrm{Q}_{e}$, for which no charge transfer takes place at impact if the particle has this initial charge. These parameters are in principle related to the contact potential difference (CPD) between contacting surfaces, as it is generally regarded as a driving force of the charge transfer. Direct measurements of the CPD of insulating powder bed and their correlations with the electrification of powders have been attempted by Yoshida et al. (1994), Itakura et al. (1996), Tanoue et al. (2001) and Matsusaka et al. (2003). It would be of great interest to explore the correlation between the CPD, as an electrical property of bulk powders, and the two parameters $\Delta \mathrm{q}_{\mathrm{o}}$ and $\mathrm{Q}_{\mathrm{e}}$ as single particle triboelectrification properties.

In this work, in order to gain a better understanding of the triboelectrification and to develop a method for characterising the charging propensity of a number of pharmaceutical powders, impact charging tests have 
been carried out with model pharmaceutical particles. In this paper, the impact charge $\Delta \mathrm{q}_{\mathrm{o}}$ is described as a function of the maximum contact area that develops during impact between a particle and a metal surface. The latter is estimated based on an elastic-plastic deformation model which takes account of the mechanical properties of the particle. The equilibrium charge $\mathrm{Q}_{\mathrm{e}}$ is related to the CPD and triboelectric series of the sample powders that are obtained by bulk powder tests.

\section{Materials and methods}

\subsection{Sample powders}

Sample particles used in this study were 1) $\alpha$-lactose monohydrate (aLM: $\mathrm{BDH}$ ) as a widely used excipient in pharmaceutical industry, 2) aspirin (ASP: acetylsalicylic acid, Sigma-Aldrich-Fluka) as a common drug, 3) sugar granules (SG: mainly sucrose, NP Pharm) as a core material for composite drugs, 4) ethylcellulose granules (EC: Hercules) as a tablet binder. All samples were sieved and the particles with a size in the range from 500 to 600 $\mu \mathrm{m}$ were used for the tests. With the present set up it is difficult to test particles smaller than $100 \mu \mathrm{m}$, because the particle velocity cannot be reliably measured due to the light detectors' limitations. This shortcoming can be overcome using Laser Doppler Anemometry. Furthermore the measurement of the electrostatic charge is also likely to be affected by noise for small particles, but this limit is yet to be identified.

\subsection{Impact charging tests}

Figure 1 shows a schematic diagram of the impact charging rig for single particles as described by Watanabe et al. (2006). In order to impart an initial 
charge to a sample particle, it is shaken for a while within a container made of metal or insulator. The charged particle is subsequently fed into the funnel at the top of the rig, and is accelerated due to gravity and downward air flow through a glass tube, and then is impacted at a stainless steel target situated in a collection chamber. The velocity of the particle is measured by a photo-detector when the particle passes through the detector prior to impact.

A through-type Faraday cage (FC1) is installed just above the photo-detector to measure the particle charge before impact (called the "initial charge" in this paper). The second Faraday cage (FC2) is installed within the collection chamber to measure the particle charge after impact. The particle impacted at the stainless steel target rebounds, and is eventually trapped on a sieve mesh located at the bottom of the inner cage of FC2. The amount of charge transfer due to the impact is quantified by subtracting the initial charge (measured by FC1) from the particle charge after impact (FC2). This is termed the "impact charge" in this paper. The impact charging tests with the sample powders were carried out at ambient conditions: room temperature (RT) and relative humidity (RH) were around $20 \sim 25{ }^{\circ} \mathrm{C}$ and $20 \sim 35 \%$, respectively.

\subsection{Charging test of bulk powders}

One gram of a powder sample was shaken manually within a container made of either glass (GS), polytetrafluoroethylene (PTFE), polypropylene (PP) or stainless steel (SS) for about two minutes, and then the charge of the powder was measured by a Faraday cage with an electrometer (Keithley 6514). The tests were repeated at least 5 times in order to make sure of a reproducible value. The tests were carried out at RT $24 \sim 27{ }^{\circ} \mathrm{C}, \mathrm{RH} 50 \sim 65 \%$. 


\subsection{Mechanical properties of the sample powders}

Mechanical properties of the sample powders were measured by a nano-indentation tester (Micro Materials Ltd.) (Ghadiri, 2006) for estimating contact area at impact. Table 1 shows Young's modulus of elasticity E and yield stress $\mathrm{Y}$ of the samples, which is obtained as one third of the indentation hardness measured by the indentation tests (Ghadiri, 2006).

\subsection{Contact potential difference of the sample powders}

The contact potential difference (CPD) was measured by a system that has been developed by Yoshida et al. (1994) and Itakura et al. (1996). The principle of the CPD measurement is essentially based on the method proposed by Lord Kelvin (1898) and Zisman (1932). However the system is specially designed for powder materials. The surface potential of a sample powder is measured against a gold-plated electrode continuously. It decays with time and eventually reaches a terminal value when the electrostatic charge on the powder bed is completely dissipated, which corresponds to the CPD of the powder against gold. The measurements were carried out at $25 \pm 1{ }^{\circ} \mathrm{C}$ and $\mathrm{RH}$ $38 \pm 5 \%$ apart from EC which was done at a RH of $60 \pm 5 \%$. Table 2 shows the CPD of the sample powders and a stainless steel sample, which is prepared as same as the target used in impact charging tests. The CPD values were originally measured with reference to gold (shown in the middle column in the table). They are converted to ones with respect to a stainless steel target (shown in the right column) by subtracting the CPD of the stainless steel (SS) vs. gold. 


\section{Results and discussion}

\subsection{Relationship between impact charge and initial charge}

The previous work showed that a linear relationship between the impact charge and the initial charge prevailed with some data scatter presumably due to variation in contact geometry due to particle shape (Watanabe et al., 2006). A schematic illustration of the linear relationships is shown in Figure 2. The impact charge at zero initial charge ( $\Delta \mathrm{q}_{\mathrm{o}}$, i.e. the intercept on the vertical axis) is considered as a characteristic charge and it increases with the impact velocity. In contrast, the intercept on the horizontal axis does not depend on the impact velocity. Therefore, this initial charge can be considered as an equilibrium charge $\mathrm{Q}_{\mathrm{e}}$, which means that no net charge transfer takes place at impact if the particle has this initial charge. For a linear dependency of the impact charge on the initial charge, the characterisation of $\Delta q_{o}$ and $Q_{e}$ is essential for establishing the charging tendency of the sample particles, and will be discussed in detail in the following sections.

\subsection{Impact charge $\Delta \mathbf{q}_{\mathrm{o}}$}

Figure 3 shows the relationship between the impact charge $\Delta q_{o}$ and the normal component of impact velocity $\mathrm{V}_{\text {in }}$. Each plot corresponds to an experimental condition for impact charging tests, such as an impact velocity and an impact angle ( $30^{\circ}$ or $60^{\circ}$ with respect to the target surface). The error bars indicate the standard error, which is deduced by the least-squares method for fitting data of the impact charges against the initial charges. For all the sample powders the impact charge increases with increasing the impact velocity. The data of three samples, i.e. SG, aLM and ASP, seem to lie on a straight line for each sample, implying that the impact charge is proportional to the normal 
component of impact velocity regardless of the impact angle. In contrast, for the case of EC, an increase in the tangential component of impact velocity (corresponding to a decrease in impact angle) makes the impact charge larger. The previous work of Matsusaka et al. (2001) for a spherical rubber ball showed that the impact charge was a function of contact area. It is, therefore necessary to estimate the contact area at impact between a particle and a target surface in order to discern the trend of the data shown in the Fig.3 more clearly.

\subsection{Estimation of contact area}

The contact mechanics for impact of non-spherical particles as used in this study is complex and requires a numerical analysis of the contact deformation, which is beyond the scope of this paper. Therefore, the contact area was estimated based on the spherical particle shape as described below.

The contact area during an elastic impact between a spherical particle and a plane is given by Hertz analysis (Timoshenko and Goodier, 1970). The elastic limit gives the yield velocity, $\mathrm{V}_{\mathrm{y}}$, which is the normal component of impact velocity below which the deformation is assumed to be elastic and is expressed as (Thornton and Ning, 1998):

$$
\mathrm{V}_{\mathrm{y}}=1.56 \sqrt{\frac{\mathrm{k}^{4} \mathrm{Y}^{5}}{\rho}}
$$

where $\mathrm{Y}$ and $\rho$ are yield pressure and density of the particle, respectively. Parameter $\mathrm{k}$ is an inverse of reduced modulus of elasticity and is expressed as:

$$
\mathrm{k}=\frac{1-v_{1}^{2}}{\mathrm{E}_{1}}+\frac{1-v_{2}^{2}}{\mathrm{E}_{2}},
$$

where $v$ is Poisson's ratio, E is Young's modulus, and subscripts 1, 2 indicate the particle and the plane, respectively. Assuming $E_{2}>E_{1}$, the second term 
in the equation can be eliminated. The yield velocity $V_{y}$ was calculated from Eq. 1 using the measured mechanical properties of the sample particles shown in Table 1 and assuming $\rho=1500 \mathrm{~kg} / \mathrm{m}^{3}$ and $v_{1}=0.3$. There are several other correlations in the literature for the yield velocity in the case of elastic-plastic deformation; see for example Johnson (1985). However, there is little numerical difference in the values of the yield velocity between the prediction of Eq. [1] and the other cited papers for the materials tested here. For simplicity, Eq. [1] is used here. The results of $\mathrm{V}_{\mathrm{y}}$ are found to be $0.8 \mathrm{~m} / \mathrm{s}$ for EC, $0.02 \mathrm{~m} / \mathrm{s}$ for ASP, $0.04 \mathrm{~m} / \mathrm{s}$ for aLM and $0.6 \mathrm{~m} / \mathrm{s}$ for SG, which are much lower than the range of the velocities in impact charging tests (as given on the horizontal axis shown in Fig. 3). Above the elastic limit, if the plastic deformation is assumed to occur under a constant yield pressure (Bitter, 1962; Masuda et al. 1976), the maximum contact area due to elastic-plastic deformation during the impact is given by (Masuda and Iinoya, 1978)

$$
\mathrm{S}=0.41 \pi \mathrm{D}_{\mathrm{p}}^{2} \sqrt{\frac{\rho}{\mathrm{Y}}}\left[\mathrm{V}_{\text {in }}-0.048 \sqrt{\frac{\mathrm{k}^{4} \mathrm{Y}^{5}}{\rho}}\right], \quad \text { at } \mathrm{V}_{\text {in }}>\mathrm{V}_{\mathrm{y}}
$$

where $D_{p}$ is diameter of the particle assumed as $550 \mu \mathrm{m}$ in this study, and $V_{\text {in }}$ is the normal component of the impact velocity. The contact area estimated from Eq.3 is linear with respect to the normal velocity above $V_{y}$, as shown in Figure 4. Obviously softer materials such EC and ASP produce larger contact areas than harder materials such aLM and SG.

Figure 5 shows the impact charge $\Delta \mathrm{q}_{\mathrm{o}}$ (previously shown in Fig.3) as a function of the estimated contact area $\mathrm{S}$ (shown in Fig.4). The values of $\Delta \mathrm{q}_{\mathrm{o}}$ for SG, aLM and ASP vary linearly with the estimated contact area. This is consistent with the previous work on charge transfer during normal impact of a spherical elastic rubber ball (Matsusaka et al., 2000). The data of the three 
samples seem to be unified with the contact area. In contrast, for the case of EC, the data does depend on impact angle. The trend is similar to that of the previous study of impact charging tests for spherical polymer particles of a few millimetres in diameter (Matsuyama and Yamamoto, 1994). In this study, the contact area was actually measured; it had an ellipsoidal shape due to the tangential component of the velocity, suggesting sliding and/or rolling of the particle at impact onto a metal plate. In contrast, no effect of the tangential component of the velocity on the impact charge was found for the cases of spherical PMMA and Nylon 66 particles of a few millimetres in diameter (Masui and Murata, 1983). Therefore, the effects of the tangential component of the impact velocity as well as the reasons why has an effect for some materials and not effect for the others are questions to be addressed in future work.

For further discussions on impact charge $\Delta \mathrm{q}_{\mathrm{o}}$, there might be other factors that need to be taken into account. The values of CPD differ among the sample particles as shown in Table 2, and the impact time should also depend on the impact velocity (Matsuyama and Yamamoto, 1994; Matsusaka et al., 2000). The contact area presented in this paper is calculated by assuming a spherical particle shape. Actually the sample particles apart from SG have non-spherical shapes such as tomahawk for aLM, oblong for ASP, irregular with quite rough surfaces for EC. For non-spherical particles, the contact area highly depends on an orientation at impact. Therefore particle shape is also a factor that should be taken into account for future work.

The surface charge density generated by a single impact is quantified by $\Delta \mathrm{q}_{\mathrm{o}} / \mathrm{S}$ for zero initial charge, where $\mathrm{S}$ is the estimated contact area. From the data shown in Fig. 5 , the charge density $\Delta q_{o} / S$ for the sample particles is 
found to be around $10^{-4} \mathrm{C} / \mathrm{m}^{2}$, which is in the same order of magnitude found in the previous studies for insulating materials (Masui and Murata, 1983; Matsuyama and Yamamoto, 1994, 1997). This suggests that the pharmaceutical powders used here are insulating materials, and hence have a high electrostatic charging propensity.

\subsection{Equilibrium charge $Q_{e}$}

Figure 6 shows the equilibrium charge, $Q_{e}$, which is defined as the intercept on the horizontal axis in the linear relationship between the impact charge and initial charge shown in Fig. 2, as a function of impact velocity. Error bars indicate the standard errors deduced by the least-squares method for fitting the data of impact charges with respect to initial charges. Although some fluctuations are seen especially in ASP, originating from a wide scatter in the data, $Q_{e}$ is obviously independent of the impact velocity and impact angle. This indicates that $Q_{e}$ does not depend on the operational conditions and should depend on the material properties of the particle and target. Therefore, the equilibrium charge $\mathrm{Q}_{\mathrm{e}}$ is considered to be a parameter expressing the charging propensity of a material. In the following sections, the equilibrium charge will be considered in terms of the triboelectric series and related to the contact potential difference of the sample powders obtained form bulk powder tests.

\subsection{Triboelectric series}

Table 3 shows the charge-to-mass ratio data of the sample powders after shaking within various containers, which are made of either glass (GS), polytetrafluoroethylene (PTFE), polypropylene (PP) or stainless steel (SS). The data shown in the table are average values from tests repeated at least 5 
times. The absolute values of the data cannot be simply compared to each other, since size and geometry of containers and shaking intensity by hand-shaking are not consistent throughout the tests. The polarity of the charge is however the essential information, and gives the so-called “triboelectric series” (Harper, 1967) as shown in Table 4. The results indicate that EC tends to be charged positively, ASP tends to be charged negatively, and SG and aLM are intermediate of the others and have a similar tendency. This order qualitatively agrees with the values of equilibrium charge $Q_{e}$, which is also shown in Table 4. Namely, for the Qe with respect to stainless steel (SS), EC has positive value, SG and aLM have a negative and similar value, and ASP has the most negative value. In general the triboelectric series is not quantitative and hence its use is limited. Generally, this agreement implies that $\mathrm{Q}_{\mathrm{e}}$ would certainly provide a better indication of the charging propensity of the particles.

The relative humidity $(\mathrm{RH})$ during the charging tests by shaking varied between 50-65 \%. However this variation did not greatly affect the charge-to-mass ratio. Rowley and Mackin (2003) investigated the effect of moisture sorption propensity on the tribo-electrification of pharmaceutical excipients. Their results showed that tribocharging of $\alpha$-lactose monohydrate was not affected by varying humidity (RH 0-80\%), since the moisture content of lactose was very insensitive to humidity. For materials more sensitive to humidity, tests have to be done under controlled humidity conditions.

\subsection{Contact potential difference}

Figure 7 shows $Q_{e}$ as a function of the contact potential difference (CPD) of the sample powders. The upper horizontal axis indicates the CPD that was 
originally measured with reference to gold, and the lower one indicates the converted CPD with respect to the stainless steel target SS, as shown in Table 2. The values of $Q_{e}$ are qualitatively correlated with the CPD vs SS. Although the CPD is considered as the driving force of the charge transfer, surprisingly the data do not go through the origin, and are not perfectly linear with respect to the CPD. There should be other factors that could not be taken into account at this stage. One possibility is an uncertainty in deducing the $\mathrm{Q}_{\mathrm{e}}$ from the data with a wide scatter (Watanabe et al., 2006). Another is the difference in the particle shape among the samples. The particle shape could affect the value of $Q_{e}$, since $Q_{e}$ is a function of particle size, i.e. essentially the surface area of the particle (Matsuyama and Yamamoto, 1997). Nevertheless the Qe obtained from single particle tests should in principle be associated with the CPD of the powders measured as a bulk powder property. To develop a better correlation between $\mathrm{Q}_{e}$ and $\mathrm{CPD}$, the single impact charging tests have to be done with different particle sizes, especially smaller ones. The range of particle sizes used in this study $(500-600 \mu \mathrm{m})$, albeit smaller than most of previous studies, it is still one order of magnitude larger than typical pharmaceutical powders. As described in section 2.1, sensitivities of charge and particle velocity measurements need to be improved to be able to test particles of tens of micrometre in size.

For future work, in order to predict particle charging in actual processes, assembly calculations are required in which the single particle charging behaviour is coupled with a hydrodynamic model of bulk flow. Recently Matsusaka and Masuda (2006) analysed the charge transfer in pneumatic conveying lines, taking account of mirror charges as well as space charge effects. As these effects are the consequences of the electrostatic 
charge on each single particle, the information obtained from the single impact charging tests conducted in this study can be coupled with the assembly dynamics to predict particle charging in actual processes. New approaches based on Distinct Element Method and combined with Computational Fluid Dynamics are emerging which are fully predictive based on single particle characteristics, e.g. Watano (2006) and Lim et al. (2006).

\section{Conclusions}

Impact charging tests with single pharmaceutical particles of $\alpha$-lactose monohydrate, aspirin, sugar granules and ethylcellulose provided two important parameters: impact charge $\Delta \mathrm{q}_{\mathrm{o}}$ and equilibrium charge $\mathrm{Q}_{\mathrm{e}} . \quad$ The $\Delta \mathrm{q}_{\mathrm{o}}$ is found to be proportional to the maximum contact area at impact. The latter was estimated based on the elastic-plastic deformation, taking account of the mechanical properties of the particles. The $\mathrm{Q}_{\mathrm{e}}$ is confirmed to be independent of impact velocity and impact angle for all samples. The data trend of Qe has a qualitative agreement with the triboelectric series. The latter is obtained from bulk powder charging tests. It is found that $\mathrm{Q}_{\mathrm{e}}$ is linearly related to the contact potential difference of the sample powders. The impact charge $\Delta q_{\text {o }}$ and equilibrium charge $\mathrm{Q}_{\mathrm{e}}$ are suggested to be the essential parameters for characterizing the charging propensity of particles.

\section{Acknowledgements}

The financial support of Merck Sharp and Dohme for this project is gratefully acknowledged. The authors would like to thank Dr Chih C. Kwan for her advice on operating the nano-indentation device and Dr Louis S. Crocker, Merck Research Laboratories, for his helpful editorial suggestions. 


\section{References}

Bailey, A.G., 1984. Electrostatic phenomena during powder handling. Powder Technol. 37, 71-85.

Bailey, A.G., 1997. The inhalation and deposition of charged particles within the human lung. J. Electrostat. 42, 25-32.

Bailey, A.G., 1998. Drug delivery by inhalation of charged particles. J. Electrostat. 44, 3-10.

Balachandran, W., Machowski, W., Gaura, E., Hudson, C., 1997. Control of drug aerosol in human airways using electrostatic forces. J. Electrostat. 40-41, 579-584.

Bitter, J.G.A., 1963. A study of erosion phenomena, Part 1. Wear, 6, 5-21.

Byron, P.R., Peart, J., Staniforth, J.N., 1997. Aerosol electrostatics I: Properties of fine powders before and after aerosolization by dry powder inhalers. Pharm. Res. 14, 698-705.

Eilbeck, J., Rowley, G., Carter, P.A., Fletcher, E.J., 1999. Effect of materials of construction of pharmaceutical processing equipment and drug delivery devices on the triboelectrification of size-fractionated lactose. Pharm. Pharmacol. Commun. 5, 429-433.

Eibeck, J., Rowley, G., Carter, P.A., Fletcher, E.J., 2000. Effect of contamination of pharmaceutical equipment on powder triboelectrification. Int. J. Pharm. 195, 7-11.

Ema, A., Yasuda, D., Tanoue, K., Masuda, H., 2003. Tribo-charge and rebound characteristics of particles impact on inclined or rotating metal target. Powder Technol. 135-6, 2-13.

Ghadiri, M., 2006. Hardness, stiffness and toughness of particles. In: Powder Technology Handbook (Chap. 1.5), 3rd Ed., Taylor \& Francis. 
Grosvenor, M.P., Staniforth, J.N., 1996. The influence of water on electrostatic charge retention and dissipation in pharmaceutical compacts for powder coating. Pharm. Res. 13, 1725-1729.

Harper, W.R., 1967. Contact and frictional electrification. Oxford University Press, London.

Itakura, T., Masuda, H., Ohtsuka, C., Matsusaka, S., 1996. The contact potential difference of powder and the tribo-charge. J. Electrostat. 38, 213-226.

Johnson, K.L. 1985. Contact mechanics. Cambridge University Press.

Kelvin, Lord W.T., 1898. Contact electricity of metals. Phil. Mag. 46, 82-120.

Lim, E.W. C., Zhang Y., Wang C.-H., 2006. Effects of an electrostatic field in pneumatic conveying of granular materials through inclined and vertical pipes. Chem. Eng. Sci. 61, 7889-7908.

Masui, N., Murata, Y., 1983. Electrification of polymer particles by impact on a metal plate. Jpn. J. Appl. Phys. 22, 1057-1062.

Masuda, H., Komatsu, T., Iinoya, K., 1976. The static electrification of particles in gas-solids pipe flow. AICHE J. 22, 558-564.

Masuda, H., Iinoya, K., 1978. Electrification of particles by impact on inclined metal plates. AICHE J. 24, 950-956.

Matsusaka, S., Ghadiri, M., Masuda, H., 2001. Electrification of an elastic sphere by repeated impacts on a metal plate. J. Phys. D-Appl. Phys. 33, 2311-2319.

Matsusaka, S., Masuda, H., 2003. Electrostatics of particles. Adv. Powder. Technol. 14, 143-166.

Matsusaka, S., Masuda, H., 2006, Simultaneous measurement of mass flow rate and charge-to-mass ratio of particles in gas-solids pipe flow. Chem. 
Eng. Sci. 61, 2254-2261.

Matsuyama, T., Yamamoto, H., 1994. Charge transfer between a polymer particle and a metal plate due to impact. IEEE Trans. Ind. Appl. 30, 602-607.

Matsuyama, T., Yamamoto, H., 1995. Charge relaxation process dominates contact charging of a particle in atmospheric conditions. J. Phys. D-Appl. Phys. 28, 2418-2423.

Matsuyama, T., Yamamoto, H., 1997. Charge relaxation process dominates constant charging of a particle in atmospheric conditions II: General model. J. Phys. D-Appl. Phys. 30, 2170-2175.

Matsuyama, T., Ogu, M., Yamamoto, H., Marijnissen, J.C.M., Scarlett, B., 2003. Impact charging experiments with single particles of hundred micrometre size. Powder Technol. 135-6, 14-22.

Matsuyama, T., Yamamoto, H., 2006. Impact charging of particulate materials, Chem. Eng. Sci. 61, 2230-2238.

Murtomaa, M., Laine, E., 2000. Electrostatic measurements on lactose-glucose mixtures. J. Electrostat. 48, 155-162.

Murtomaa, M., Ojanen, K., Laine, E., 2002. Effect of surface coverage of a glass pipe by small particles on the triboelectrification of glucose powder. J. Electrostat. 54, 311-320.

Murtomaa, M., Mellin, V., Harjunen, P., Lankinen, T., Laine, E., Lehto, V.P., 2004. Effect of particle morphology on the triboelectrification in dry powder inhalers. Int. J. Pharm. 282, 107-114.

Rowley, G., 2001. Quantifying electrostatic interactions in pharmaceutical solid systems. Int. J. Pharm. 227, 47-55

Rowley, G., Mackin, L.A., 2003. The effect of moisture sorption on 
electrostatic charging of selected pharmaceutical excipient powders. Powder Technol. 135-136, 50-58.

Staniforth, J.N., Rees, J.E., 1981a. Electrostatic charge interactions in ordered powder mixes. J. Pharm. Pharmacol. 34, 69-76.

Staniforth, J.N., Rees, J.E., 1981b. Powder mixing by tribo-electrification. Powder Technol. 30, 255-256.

Stainforth, J.N., 1994. The importance of electrostatic measurements in aerosol formulation and reformulation. Respiratory Drug Delivery IV, 303-311.

Tanoue, K., Mrita, K., Maruyama, H., Masuda, H., 2001. Influence of functional group on the electrification of organic pigments. AICHE J. 47, 2419-2424.

Thornton, C., Ning, Z., 1988. A theoretical model for the stick/bounce behaviour of adhesive, elastic-plastic spheres. Powder Technol. 99, 154-162.

Timoshenko, S.P., Goodier, J.N., 1970. Theory of elasticity. 3rd Ed., McGraw-Hill, New York.

Watanabe, H., Samimi, A., Ding, Y.L., Ghadiri, M., Matsuyama, T., Pitt, K.G., 2006. Measurement of charge transfer due to single particle impact, Part. Part. Syst. Charact. 23, 1-5.

Watano S., 2006. Mechanism and control of electrification in pneumatic conveying of powders. Chem. Eng. Sci. 61, 2271-2278.

Yamamoto, H., Scarlett, B., 1986. Triboelectric charging of polymer particles by impact. Part. Part. Syst. Charact. 3, 117-121.

Yoshida, H., Iguchi, Y., Matsumoto, T., Masuda, H., Murata, H., Yamada, Y., 1994. Effect of surface coating ratio of surface-modified particles on the contact potential difference between a powder bed and a metal. Adv. 
Powder Technol. 5, 377-391.

Zisman, W.A. 1932. A new method of measuring contact potential differences in metals. Rev. Sci. Instrum. 3, 367-368 


\section{Captions of tables and figures}

Table 1 Mechanical properties of the sample particles measured by nano-indentation tests

Table 2 Contact potential difference of the sample powders

Table 3 Summarised results of bulk charging tests

Table 4 Triboelectric series and equilibrium charge

Figure 1 A Schematic diagram of impact charging test rig for single particles

Figure 2 A schematic illustration of the linear relationships between impact charge and initial charge based on the results of spherical sugar granules (impact angle: $30^{\circ}$ )

Figure 3 Impact charge $\Delta \mathrm{q}_{\mathrm{o}}$ as a function of the normal component of impact velocity $\mathrm{V}_{\text {in }}$

Figure 4 Estimated contact area $\mathrm{S}$ as a function of the normal component of impact velocity $\mathrm{V}_{\text {in }}$

Figure 5 Impact charge $\Delta \mathrm{q}_{\mathrm{o}}$ as a function of the contact area $\mathrm{S}$

Figure 6 Equilibrium charge $\mathrm{Q}_{\mathrm{e}}$ as a function of the impact velocity $\mathrm{V}_{\mathrm{i}}$

Figure 7 Plot of the equilibrium charge $Q_{e}$ against contact potential difference (CPD) of the sample powders 
Table 1

\begin{tabular}{lcc}
\hline Sample & E [GPa] & Y [MPa] \\
\hline Sugar granules, SG & 23 & 600 \\
$\alpha$-lactose, aLM & 18 & 170 \\
Asprin, ASP & 7.2 & 70 \\
Ethlycellulose, EC & 1.1 & 60 \\
\hline
\end{tabular}

Table 2

\begin{tabular}{lcc}
\hline Sample & CPD [V] vs. Au & CPD [V] vs. target \\
\hline Ethlycellulose, EC & 0.37 & 0.25 \\
$\alpha$-lactose, aLM & 0.27 & 0.15 \\
Sugar granules, SG & 0.14 & 0.02 \\
Aspirin, ASP & -0.23 & -0.35 \\
\hline Stainless steel & 0.12 & - \\
\hline
\end{tabular}


Table 3

\begin{tabular}{c|cccc}
\hline \multirow{2}{*}{ Sample } & \multicolumn{4}{|c}{ Material of containers } \\
\cline { 2 - 5 } & GS & SS & PP & PTFE \\
\cline { 2 - 5 } & \multicolumn{4}{|c}{ Charge-to-mass ratio, nC/g } \\
\hline \hline EC & +29.0 & +21.7 & +51.0 & +44.6 \\
\hline aLM & -3.73 & -0.021 & +13.1 & +23.4 \\
\hline SG & -3.55 & -0.024 & +10.7 & +30.5 \\
\hline ASP & -4.04 & -0.128 & -8.9 & +9.4 \\
\hline
\end{tabular}

Table 4

\begin{tabular}{cc}
\hline Positive end & Qe vs. SS [pC] \\
\hline EC & +10 \\
GS, $\underline{\text { SS }}$ & $-18,-16$ \\
aLM, SG & -40 \\
PP & \\
ASP & \\
PTFE & \\
\hline Negative end
\end{tabular}


Fig. 1

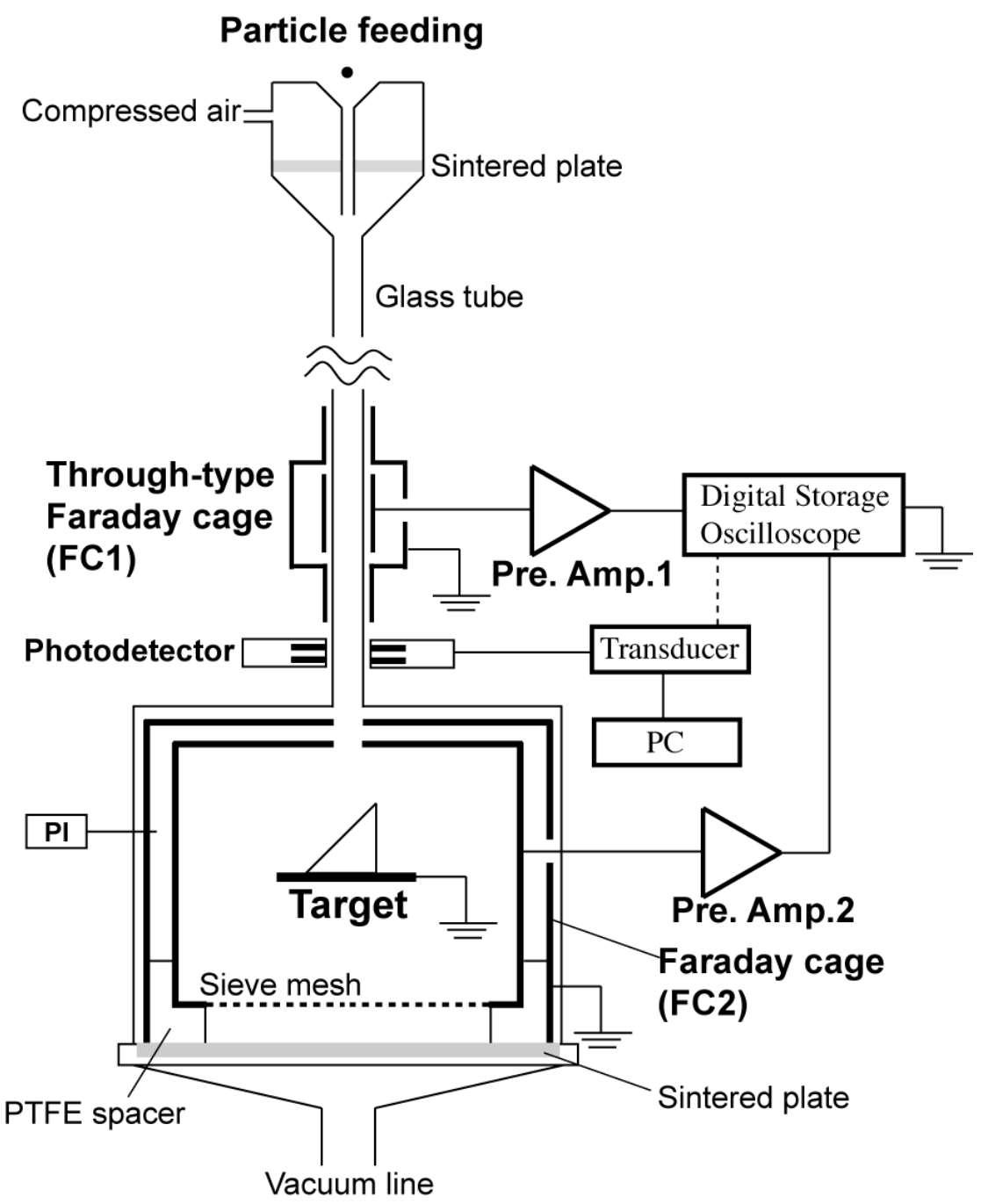


Fig. 2

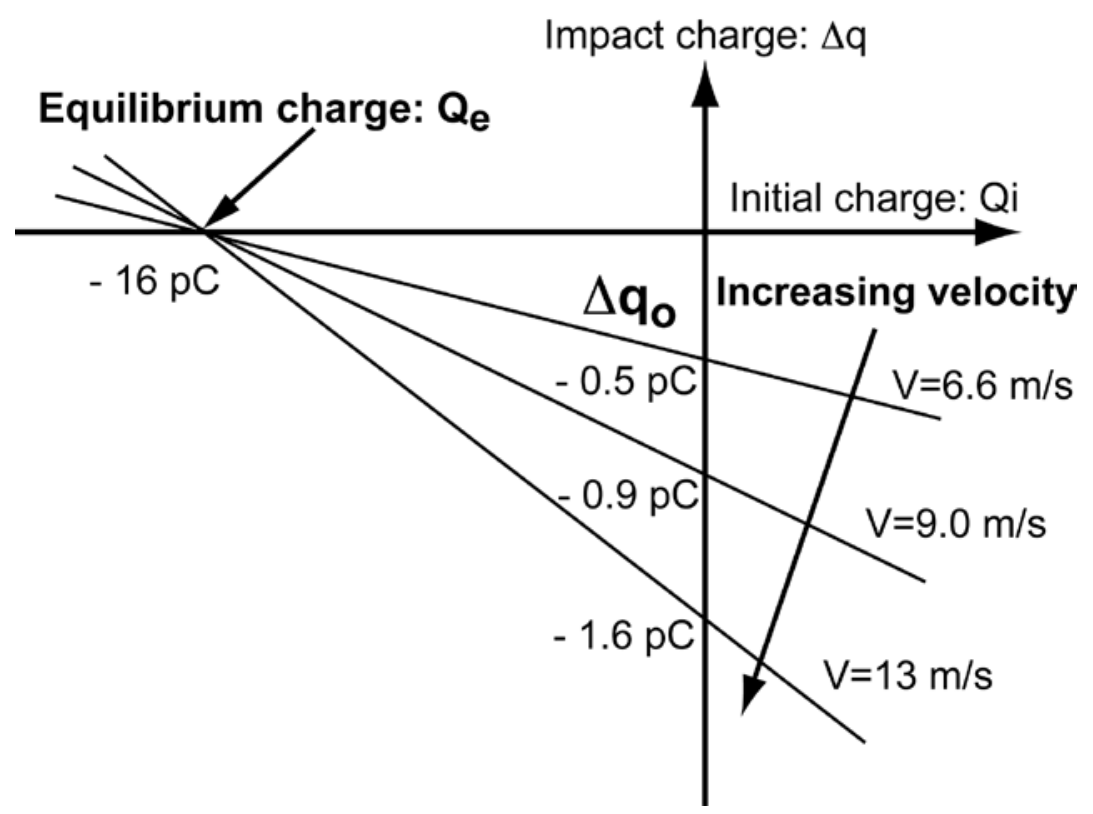


Fig. 3

Normal component of impact velocity, $V_{\text {in }}[\mathrm{m} / \mathrm{s}$ ]

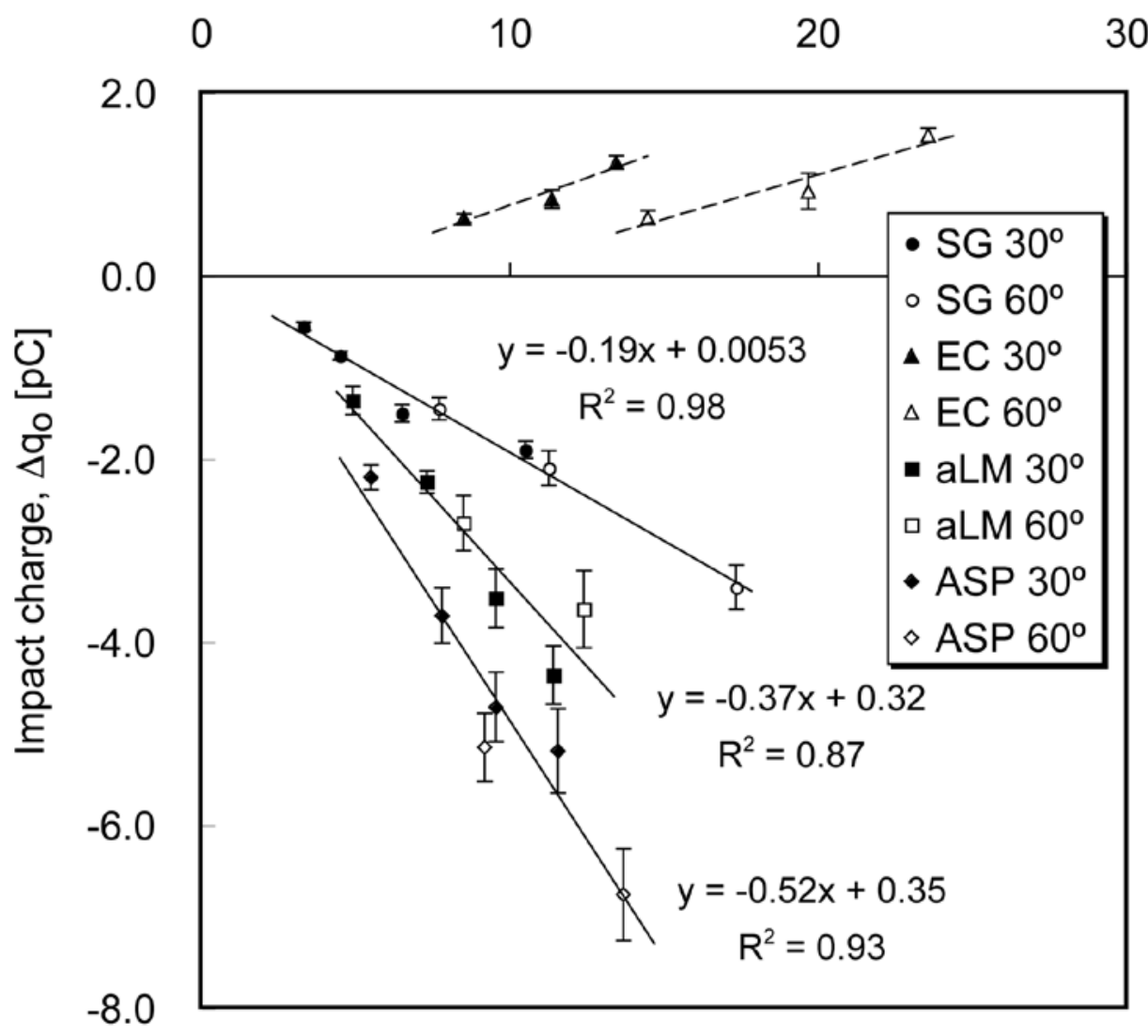


Fig.4

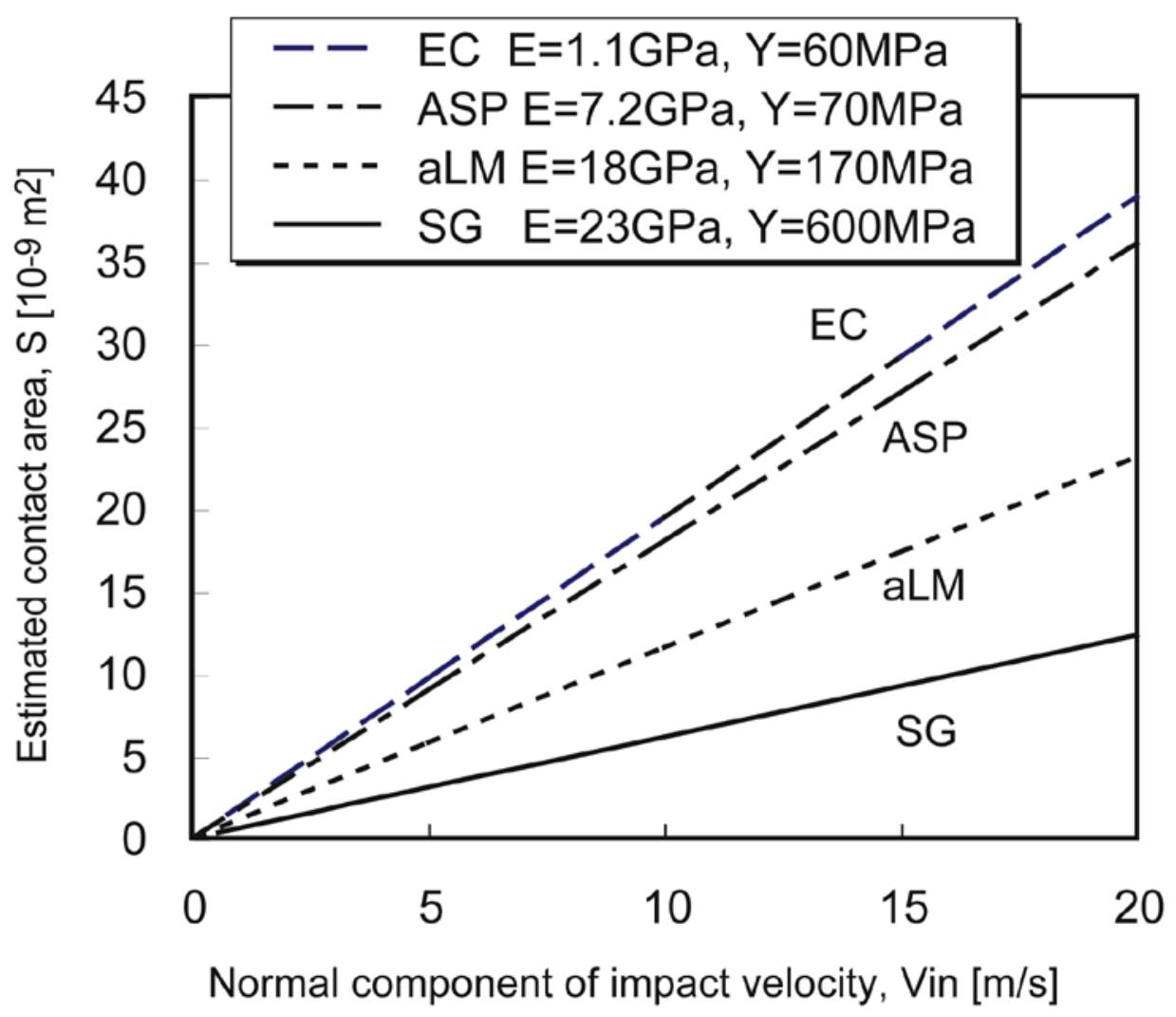


Fig.5

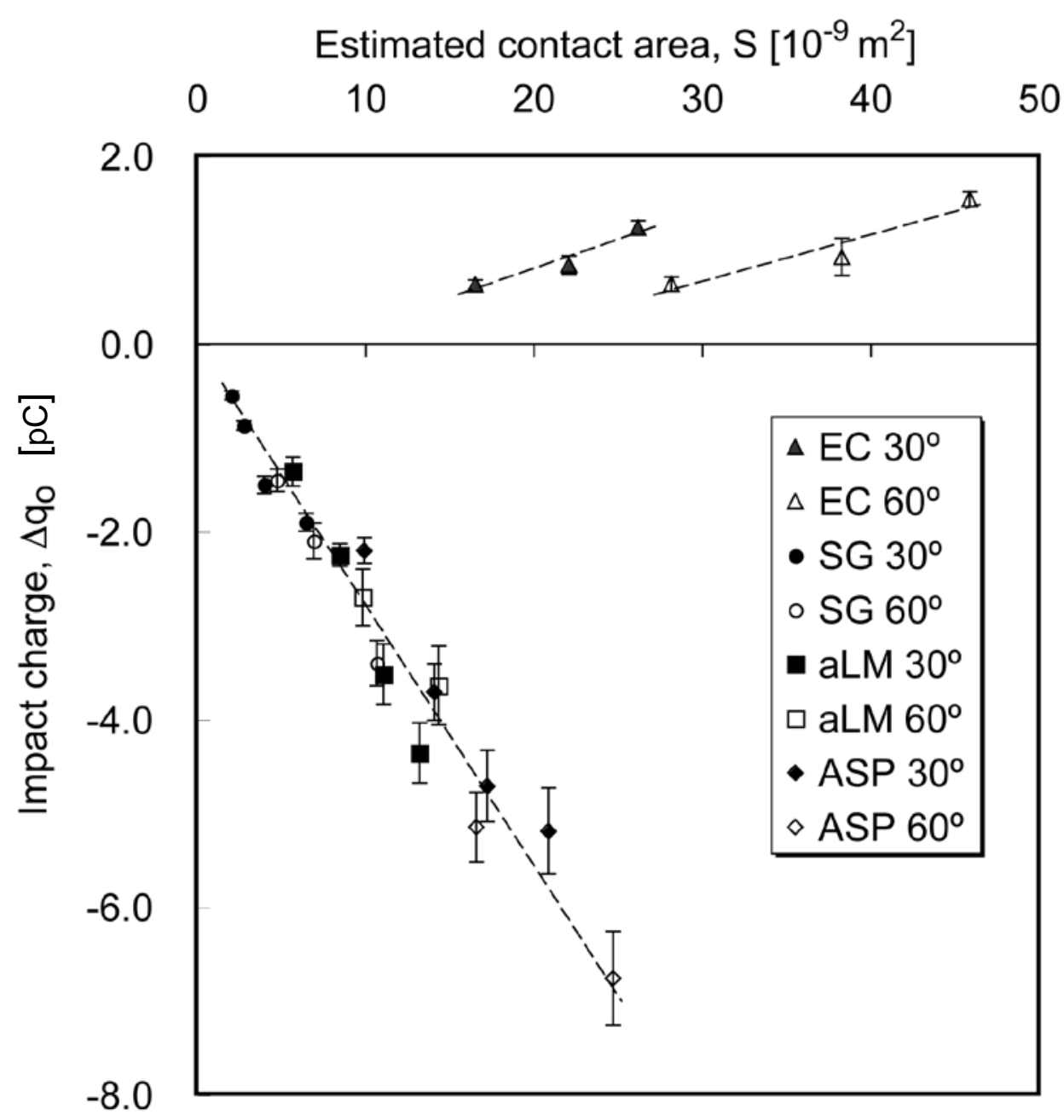


Fig.6

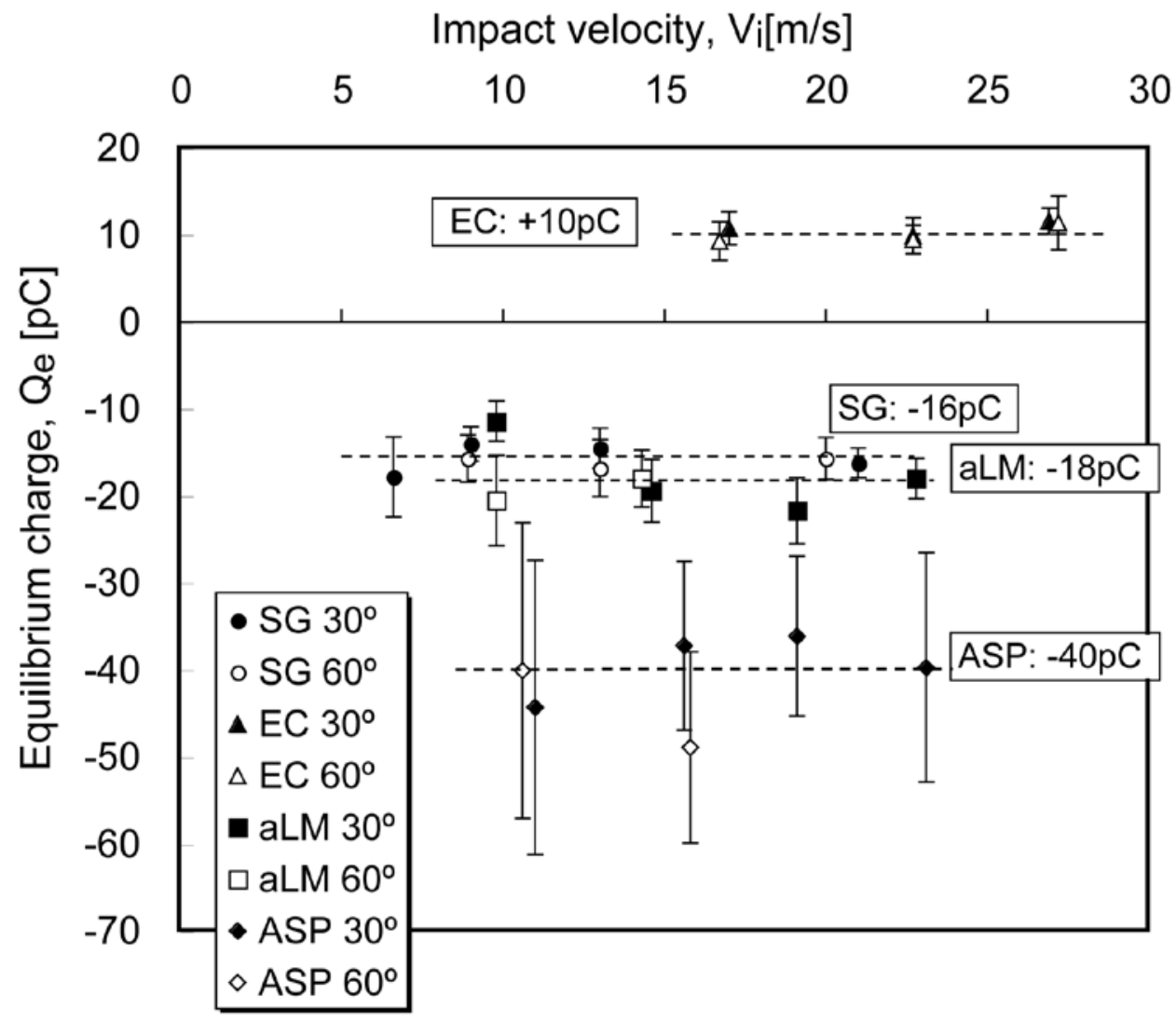


Fig.7

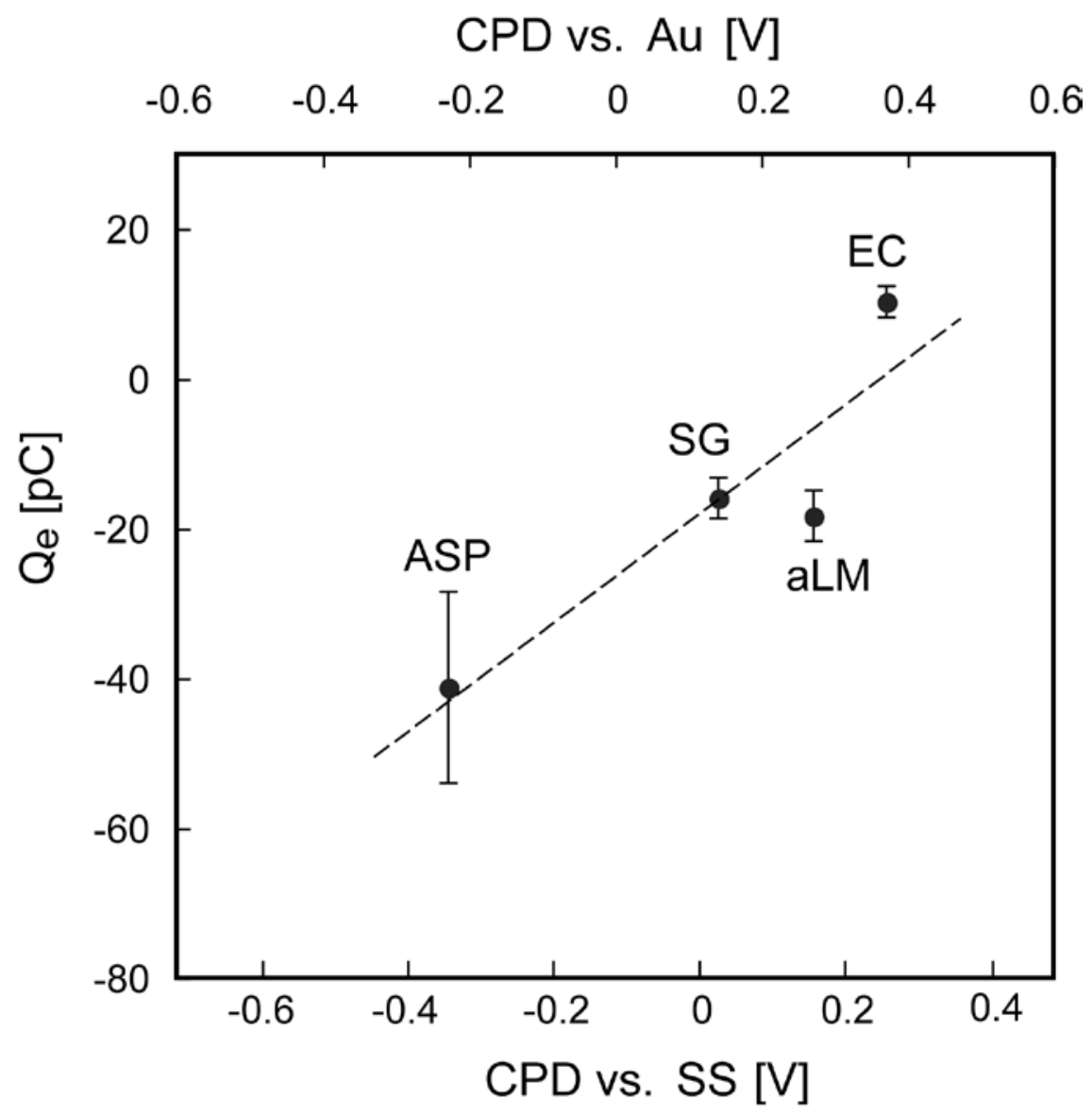

